\title{
Multifunctional Thermally Remendable Nanocomposites
}

\author{
Edward D. Sosa, ${ }^{1}$ Thomas K. Darlington, ${ }^{2}$ Brian A. Hanos, ${ }^{2}$ and Mary Jane E. O'Rourke ${ }^{3}$ \\ ${ }^{1}$ ERC Inc., 2224 Bay Area Boulevard, Houston, TX 77058, USA \\ 2 nanoComposix, 4878 Ronson Ct., San Diego, CA 92111, USA \\ ${ }^{3}$ NASA Johnson Space Center, 2101 NASA Parkway, Houston, TX 77058, USA
}

Correspondence should be addressed to Edward D. Sosa; edward.sosa-1@nasa.gov

Received 7 May 2014; Accepted 20 June 2014; Published 7 July 2014

Academic Editor: Yuanxin Zhou

Copyright (C) 2014 Edward D. Sosa et al. This is an open access article distributed under the Creative Commons Attribution License, which permits unrestricted use, distribution, and reproduction in any medium, provided the original work is properly cited.

\begin{abstract}
Challenges associated with damage tolerance in polymer matrix composites must be successfully addressed in order to ensure highly reliable structures with significant weight savings. Self-healing materials provide a viable means to surmount damage tolerance concerns, thereby allowing for the realization of the mass reduction such structures have promised but not yet achieved. Introduction of multifunctional properties into self-healing composites can further extend their usefulness. This study examines the incorporation of carbon nanotubes into a self-healing composite in order to achieve this. Composite panels were fabricated with carbon fibers, a bismaleimide tetrafuran (2MEP4F) polymer resin, and various carbon nanotube materials. The composites exhibit enhancement in electrical, mechanical, and thermal properties. The healing mechanism is a thermally activated reversible polymerization of the $2 \mathrm{MEP} 4 \mathrm{~F}$ resin. The proposed method of heating exploits the enhanced microwave absorption inherent to carbon nanotubes to provide the thermal energy required for the reversible polymerization. Microwave testing demonstrated that the heating efficiency is increased, allowing uniform heating to the required temperature for polymer healing. Impacted composites show localized heating at the damage site, which implies that microwave heating can also be used as a means for damage detection and potential structural health monitoring.
\end{abstract}

\section{Introduction}

The National Aeronautics and Space Administration (NASA) is currently evaluating composite materials for primary and secondary structures in habitat modules, crew vehicles, pressure vessels, and other potential applications. Lighter-weight materials with high specific strength can lead to drastic reductions in uptake mass, resulting in more cost effective space exploration. Such materials can have similar benefits in commercial applications such as aircrafts, vehicles, and wind turbine blades, as well as a variety of infrastructural applications. As such they are of great interest not only to NASA but also to the commercial sector. Polymer matrix composites have attracted much attention due to their relatively high strength, light weight, and low cost. An integral design concern, however, is that of damage tolerance. Nearly imperceptible cracks may form upon impact which, while being microscopic, may have drastic effects on structural integrity. As a result, structural composites are designed to be thicker and heavier than would otherwise be required, thus negating some of the weight savings they promise. One way to compensate for this concern is to design advanced materials capable of self-repair. Collaborative work between the NASA Johnson Space Center and nanoComposix is aimed at the development of a multifunctional, damagetolerant nanocomposite that utilizes carbon nanotubes and a thermally healable polymer resin.

There are many documented mechanisms of self-healing in composite materials [1-3]. Largely, they may be categorized into one of two types: (1) the inclusion of storage vessels containing the healing agent or (2) the use of a resin system which exhibits an intrinsic chemical property that can facilitate healing. To date, the most promising and successful healable materials have utilized hollow fibers and microcapsules infused with a healing agent or a polymer system capable of reversible polymerization. The infused 
storage vessels result in a truly autonomous system since the propagating crack imparts sufficient energy to rupture the vessels and the contained liquid seeps out and seals the crack [4-6]. These vessels can be filled with fluorescent markers in addition to resin, thus providing a means of structural health monitoring [4]. While the autonomous nature of this system is highly desirable, there are some disadvantages. These include the lack of multihealing capability in a single location, the reduction of structural integrity of the matrix with high loadings, the requirement of proper mixing of resin and curing agents, and limited manufacturing of complex parts since processing methods could potentially rupture the vessels. The reversible polymerization approach, while it may not be considered completely autonomous, circumvents the disadvantages of the storage vessel model. In the reversible polymerization approach, an input of energy is required by the system to initiate dissociation of chemical bonds, allowing the material to flow and seal the crack. Upon removal of the energy, bonds reform and cross-linking is restored within the matrix. The energy for healing can be supplied in the form of thermal or electromagnetic energy. Approaches to reversible polymerization have involved photo-induced reversible polymerization [7-9] or thermal activation of the matrix [10-14]. Thermal energy can be supplied by electrical resistivity or photo-induced thermal generation. Photo-induced heating involves infrared (IR) light or microwaves typically found in flash lamps or microwave ovens. While polymers can absorb IR, thermal generation is relatively inefficient as high temperature and power is generally required. Most polymers are transparent to microwaves and those with low dielectric constants do not absorb at all [15]. Additives are therefore needed. In the case described herein, healing will be induced by the microwave absorption of carbon nanotubes embedded within the composite.

Carbon nanotubes (CNTs) have been shown to absorb microwave radiation and readily convert it to heat with extremely high efficiency [16-19]. Previous research has shown that incorporation of CNTs into matrices can heat materials beyond $500^{\circ} \mathrm{C}$ within minutes when subjected to a frequency of $2.45 \mathrm{GHz}$ [20]. The microwave absorption property has been exhibited in both raw and purified nanotubes and is attributed to electronic conduction losses [18] within the CNTs. Carbon nanotubes were introduced into the thermally reversible polymer system not only to provide a heating method to drive the healing mechanism, but also to introduce multifunctionality and potential health monitoring. Researchers have shown that when CNT/epoxy composites are damaged and resistively heated, thermal discontinuity can be monitored with infrared cameras [21]. This work is intended to demonstrate a similar capability in a self-healing composite under microwave heating.

Composite panels were fabricated by coating carbon fiber fabric with a variety of CNTs. Layered fabrics were then infiltrated with bismaleimide and tetrafuran resins through vacuum assisted resin transfer molding. The bismaleimide and tetrafuran resins undergo Diels Alder chemistry to form a bismaleimide tetrafuran polymer (2MEP4F). The CNTs incorporated in the composite panels include single wall, multiwall, and spun multiwall nanotubes. The electrical, thermal, and mechanical properties were measured to determine the multifunctionality of these composites. Panels were subjected to microwave radiation in order measure the effect of nanotube type on the heating efficiency of the composites. The temperature profiles of the composite surfaces were monitored during irradiation to establish the uniformity of the thermal distribution across the surface of the composite. It was observed that CNT incorporation influences the properties of these composites.

\section{Experimental}

Composite panels were fabricated using Hexcel plain weave HexForce carbon fabric cut in $10 \mathrm{~cm}^{2}$ sheets. The fibers were cut such that a 6-ply laminate composite could be fabricated with a $\left[0^{\circ}, 60^{\circ},-60^{\circ}\right]_{2 s}$ orientation. Prior to composite layup, the fibers were coated with the particular carbonbased nanomaterial to be included, such as multiwall/single wall carbon nanotubes (SWCNTs), or multiwall nanotube ribbons spun from nanotube forests. Nanotube powders were dispersed in methanol and deposited using a hand-held sprayer. Powder samples were dispersed using a Cole Parmer Ultrasonic Processor Model CPX 500 with a model CV33 probe. Powders were sonicated for 10 minutes with amplitude at $33 \%$ of the maximum 500 watts power. Multiwall carbon nanotube (MWCNT) solutions were prepared by dispersing $50 \mathrm{mg}$ of MWCNTs in $250 \mathrm{~mL}$ of methanol, which was found to allow for the best coverage of carbon fibers. The MWCNTs used were Mitsui nanotubes from production lots which consist of different lengths and diameters. The best coverage of SWCNTs was obtained by dispersion of $25 \mathrm{mg}$ of purified SWCNTs in $250 \mathrm{~mL}$ of methanol. The SWCNTs used were HiPco tubes obtained from Rice University and pulse laserablated tubes produced at NASA's Johnson Space Center. Both materials were purified by the soft bake method [2224]. Spun MWCNT nanoribbon (s-MWCNT) materials were procured from Solarno, Inc. Carbon fiber fabrics were provided to Solarno, Inc., who proceeded to deposit s-MWCNTs drawn from nanotube forests. Spun MWCNTs were directly laid on the fibers and adhesion was accomplished by a densification process in which methanol is sprayed over the coated fibers. The s-MWCNTs were deposited on several fiber sets with various nanotube orientations relative to the fibers. Some fiber sets were coated such that when the panels were fabricated, the s-MWCNTs were all oriented along the $0^{\circ}$ direction on the front side of every ply. Other sets were coated such that the s-MWCNTs were oriented along the $0^{\circ}$ direction on the front side and along the $90^{\circ}$ direction of the reverse side of every ply. All coated fabrics were characterized by scanning electron microscopy (SEM) to establish degree of fiber coverage prior to panel fabrication.

A total of ten composite panels with $10 \mathrm{~cm} \times 10 \mathrm{~cm}$ dimensions were fabricated using above described materials (Table 1). Panels were fabricated by infiltrating coated fibers with the $2 \mathrm{MEP} 4 \mathrm{~F}$ resin. Of the ten panels, eight were fabricated by nanoComposix through a custom resin transfer molding of the $2 \mathrm{MEP} 4 \mathrm{~F}$ resin $[10,14]$. The last two panels fabricated had SWCNTs directly dispersed into the resin system and were fabricated by a wet layup process. 
TABLE 1: Measurements of the electrical, thermal, and mechanical properties of nanoadditive containing composite panels relative to the control. Thermal conductivity values are given for the two temperature extremes over the range for which they were measured.

\begin{tabular}{|c|c|c|c|c|c|c|}
\hline \multirow{2}{*}{ Panel } & \multirow{2}{*}{ Nanoadditive } & \multirow{2}{*}{$\begin{array}{l}\text { Resistivity } \\
(\Omega / \text { square })\end{array}$} & \multicolumn{2}{|c|}{ Thermal conductivity $(\mathrm{W} / \mathrm{m} \cdot \mathrm{K})$} & \multirow{2}{*}{$\begin{array}{l}\text { Storage modulus } \\
(\mathrm{GPa}) \\
30^{\circ} \mathrm{C}\end{array}$} & \multirow{2}{*}{$\begin{array}{l}\text { Loss modulus } \\
(\mathrm{GPa}) \\
30^{\circ} \mathrm{C}\end{array}$} \\
\hline & & & $30^{\circ} \mathrm{C}$ & $110^{\circ} \mathrm{C}$ & & \\
\hline$(1)$ & Control-carbon fiber only ${ }^{*}$ & $\infty$ & 0.476 & 0.482 & 42.1 & 6.95 \\
\hline (2) & $\begin{array}{l}1 \text { ply s-MWCNT }+5 \text { plies with } \\
\text { spray-coated MWCNTs (lot } 1 \text { ) }\end{array}$ & $\infty$ & 0.544 & 0.566 & 61.5 & 10.5 \\
\hline (3) & Spray-coated MWCNTs (lot 1) & 15637 & 0.583 & 0.621 & 61.6 & 9.41 \\
\hline (4) & Spray-coated MWCNTs $(\text { lot } 1)^{* *}$ & $\infty$ & 0.609 & 0.579 & 64.9 & 9.94 \\
\hline$(5)$ & Spray-coated MWCNTs (lot 2) & 13597 & 0.544 & 0.551 & 53.0 & 11.2 \\
\hline (6) & Spray-coated purified laser SWCNTs ${ }^{* * *}$ & 1673498 & 0.549 & 0.578 & 67.0 & 9.03 \\
\hline (7) & $\begin{array}{l}\text { Unidirectional }\left(0^{\circ} \text { orientation }\right) \text { spun } \\
\text { MWCNTs on front side only }{ }^{\#}\end{array}$ & 770 & 0.687 & 0.747 & 62.3 & 8.42 \\
\hline (8) & $\begin{array}{l}\text { Unidirectional }\left(0^{\circ} \text { orientation }\right) \text { spun } \\
\text { MWCNTs on front side only }\end{array}$ & $\infty$ & 0.542 & 0.525 & 49.0 & 10.0 \\
\hline (9) & $\begin{array}{l}\text { Bidirectional spun MWCNTs with } \\
\text { purified HiPco SWCNTs dispersed in } \\
\text { resin }^{\# \#}\end{array}$ & 7342 & 0.509 & 0.447 & 48.4 & 5.02 \\
\hline (10) & $\begin{array}{l}\text { Bidirectional spun MWCNTs with } \\
\text { purified laser SWCNTs dispersed in resin }\end{array}$ & 475902 & 0.540 & 0.482 & 21.7 & 2.41 \\
\hline
\end{tabular}

${ }^{*}$ Black, ${ }^{* *}$ Brown, ${ }^{* * *}$ Orange, ${ }^{\#}$ Blue, ${ }^{\# \#}$ Purple, and ${ }^{\# \#}$ Green.

Composite panels were characterized by a variety of analytical methods to establish their electrical, thermal, mechanical, and microwave absorption properties. Surface resistivity was used to assess the electrical conductivity of composites. Thermal analysis consisted of measurement of the glass transition temperature $\left(T_{g}\right)$, heat capacity $\left(C_{p}\right)$, thermal expansion, thermal diffusivity $(\alpha)$, and thermal conductivity $(\kappa)$ of each panel. Thermal properties were measured with differential scanning calorimetry (DSC), dynamic mechanical analysis (DMA), thermal mechanical analysis (TMA), and laser flash analysis (LFA). Mechanical testing consisted of dynamic mechanical analysis (DMA) and impact testing. The microwave absorption property was determined by observation of the temperature profile during sample exposure. In order to measure these properties, each $10 \times$ $10 \mathrm{~cm}$ panel was cut into four $5 \times 5 \mathrm{~cm}$ panels. One of the smaller panels was then further reduced to the size required for thermal analysis. To ensure that cutting did not introduce any potential defects that could influence thermal measurements, the panels were inspected by flash thermography before and after cutting and found to be defect-free.

Electrical resistivity measurements were conducted on three $5 \times 5 \mathrm{~cm}$ panels using a Lucas Labs 302 resistivity probe and a Keithley 2400 source meter in a 2-point configuration. Measurements were made in three different locations on each side of the panels. Measurements were made in two corners and in the center of the panels in a diagonal pattern. The lowest value was taken as the measure of the potential improvement to the electrical conductivity as the result of nanoadditive inclusion.
Glass transition temperatures $\left(T_{g}\right)$ and heat capacities $\left(C_{p}\right)$ of composites were obtained on TA Instruments DSC Q100. Composite panel samples were cut into approximately $2 \mathrm{~mm}^{2}$ samples for measurement. $T_{g} \mathrm{~s}$ were obtained through a heat/cool/heat cycle. The samples were first cooled to $0^{\circ} \mathrm{C}$ and held there for a five-minute equilibrium period. Samples were then heated to $110^{\circ} \mathrm{C}$ at ramp rate of $5^{\circ} \mathrm{C} / \mathrm{min}$, followed by cooling to $0^{\circ} \mathrm{C}$ at $10^{\circ} \mathrm{C} / \mathrm{min}$, and then heated again to $110^{\circ} \mathrm{C}$ at $5^{\circ} / \mathrm{min}$. After the second heat cycle, the temperature was held at $110^{\circ} \mathrm{C}$ for a one-minute equilibration. The $T_{g}$ was obtained from the second temperature ramp to compensate for any curing or outgassing effects that may have influenced the value during the first cycle. $C_{p}$ was measured directly after $T_{g}$ by performing DSC in the modulated mode. The sample was cooled to $0^{\circ} \mathrm{C}$ and equilibrated for one minute followed by $3^{\circ} \mathrm{C} / \mathrm{min}$ ramp to $110^{\circ} \mathrm{C}$ concluding with a three-minute equilibration step. For each panel, three sets of measurements were taken and averaged.

Thermal expansion with respect to temperature was measured using TA Instruments TMA Q400. Composites were cut into approximately $3 \mathrm{~mm} \times 10 \mathrm{~mm}$ test specimens for adequate contact with the instrument probe. Throughthickness dimensional change was measured between 25 and $110^{\circ} \mathrm{C}$ for each sample. Samples were cooled with a water chiller to $25^{\circ} \mathrm{C}$ and held for a one-minute equilibration followed by temperature ramp at a rate of $3^{\circ} \mathrm{C} / \mathrm{min}$. Each sample was run twice to compensate for any curing effects or outgassing during measurements. Three data points were obtained and averaged.

Thermal diffusivity of the composites was measured using a Netzsch LFA457 MicroFlash system. Samples were cut into 
$10 \mathrm{~mm} \times 10 \mathrm{~mm}$ squares for through-thickness measurement. Each sample was coated with a thin film of graphite paint as required for LFA analysis. The density of each sample is calculated from sample dimensions measured with a set of calipers and weighing of the sample.

Diffusivity measurements were taken from 30 to $110^{\circ} \mathrm{C}$ in $10^{\circ} \mathrm{C}$ increments. As with all other analyses, three samples of each composite were evaluated. In LFA, three laser shots are taken on each sample at each temperature. Therefore nine data points are used to report the average value. The thermal conductivity for each composite can be calculated using

$$
\kappa(T)=\alpha(T) \cdot C_{p}(T) \cdot \rho(T) .
$$

Using the $C_{p}$ values from DSC, density change $(\Delta \rho)$ obtained by TMA, and the diffusivity $(\alpha)$ from LFA, the thermal conductivity $(\kappa)$ may be determined.

Storage modulus, loss modulus, and loss tangent for each composite were measured using TA Instruments DMA 2980. Composites were cut into approximately $10 \mathrm{~mm} \times 50 \mathrm{~mm}$ test specimens. Measurements were made in three-point bend mode, with each sample run twice, again to account for any incomplete curing or outgassing that could influence the results. Exact dimensions for each sample were measured with calibers and recorded. The temperature profile consisted of an initial equilibration at $25^{\circ} \mathrm{C}$ followed by a temperature ramp from 25 to $100^{\circ} \mathrm{C}$ at a rate of $3^{\circ} \mathrm{C} / \mathrm{min}$ with an amplitude of $15 \mu \mathrm{m}$, a preload force of $0.010 \mathrm{~N}$, and a force track of $125 \%$. For each panel, three sets of measurements were taken and averaged.

Composite samples were impacted with an IMATEK impact tester using $1.13 \mathrm{~kg}(2.5 \mathrm{lbs})$ of weight and drop heights of 5 and 10 inches, corresponding to forces of $1.15 \mathrm{~J}$ $(0.85 \mathrm{ft} \cdot \mathrm{lb})$ and $2.25 \mathrm{~J}(1.66 \mathrm{ft} \cdot \mathrm{lb})$. Following impaction, panels were inspected by flash thermography and SEM to detect damage incurred by the composite. Flash thermography was performed using a thermal wave imaging EchoTherm system. SEM images were captured in the impacted area with a Zeiss Supra 55VP microscope. A qualitative measure of the toughness was established by the degree of crack formation and crack propagation.

Since microwave absorption is the proposed heating mechanism for thermal repolymerization, the effect of nanoadditives on microwave absorption was examined. Several panels were exposed to microwaves to determine the influence of CNT addition on the heating efficiency. A total of six panels were sent to the University of Minnesota for microwave irradiation with in situ temperature monitoring. Panels were subjected to microwave exposures in a Pelco BioWave microwave system with in situ thermal imaging via a FLIR infrared camera. All panels were exposed to a microwave frequency of $2.45 \mathrm{GHz}$, while the power and exposure time was adjusted to reach the required temperature for repolymerization. The six panels exposed to microwaves consisted of panels 1, 2, 3, 5, 6, and 7 as listed in Table 1 . Two smaller $5 \times 5 \mathrm{~cm}$ panels containing embedded CNTs were also impacted and subjected to microwave irradiation.

\section{Results and Discussion}

The enhancement of the nanocomposite properties is strongly dependent on the adequate dispersion of nanomaterials throughout the composite matrix. Appropriate concentrations of the nanoadditives must be uniformly distributed within the composite. Furthermore, the interaction of the nanoadditives with the matrix and fibers will significantly affect the mechanical properties. In these particular composites, the dispersion of nanoadditives is expected to be influenced by the degree of fiber coverage, the interface adhesion between nanoadditives and fibers, and the interaction of CNTs with the polymer resin during infiltration.

Coated fiber fabrics were inspected by SEM for determination of the degree of nanomaterial coverage prior to composite fabrication. Figure 1 shows areas of fiber coating with good levels of MWCNT, SWCNT, and spun MWCNTs coverage. Some areas of the fiber fabrics exhibit better coverage than others. Complete coverage of the fiber fabrics was not accomplished as exposed fibers were evident on every fabric. The weight percent of coating differed from panel to panel ranging from $0.25 \mathrm{wt} \%$ for SWCNT-coated fabrics to $1.5 \mathrm{wt} \%$ for spun CNT coated fabrics due to the degree and method of application of the nanotubes to the fibers.

After cutting panels to smaller sizes for various interrogations, the cross-sections were examined to establish the location of CNT throughout the composite. In composites containing MWCNT-coated fabrics, a greater extent of CNT migration into the matrix is observed (Figure 2(a)). In composites with spun CNTs, the CNTs primarily remain in close proximity to the fiber (Figure 2(b)) with some migration observed. In laser SWCNT-coated fabrics, nanotubes stay in close proximity to the fibers (Figure 2(c)). The two composites fabricated with a mixture of spun CNTs and dispersed SWCNTs show a combinatory effect. The spun CNTs remain near the fiber while the dispersed SWCNTs reside as agglomerates with the resin due to dispersion of the SWCNT as agglomerates rather than as individual nanotubes (Figure $2(\mathrm{~d})$ ). The greater migration of MWCNTs into the matrix is attributed to a lower degree of entanglement due to their larger diameter and greater stiffness. It is believed that this allows them to be carried along with the resin more readily during infiltration. Spun CNTs have greater entanglement and density. This, combined with van der Waals adhesion to fibers, results in lower migration. Spun CNTs that do migrate further into the matrix are thought to be CNTs that originally resided between the tows of the fibers. As for the composite with spray-coated laser SWCNTs, the low degree of migration is attributed to stronger van der Waals interaction with the fiber due to the purity of tubes. It is clear that the distribution of CNTs within the composites varies relative to nanotube type.

Electrical conductivity of composites was established by measuring the resistivity on each side and at three different locations. It was observed that resistivity varied between the different composites from infinitely high (>10 G $\Omega /$ square) to a several $\mathrm{k} \Omega$ /square. The lowest measured value for each sample is reported in Table 1 . The control exceeds a resistivity $10 \mathrm{G} \Omega$ /square in all measured locations as expected. Even 


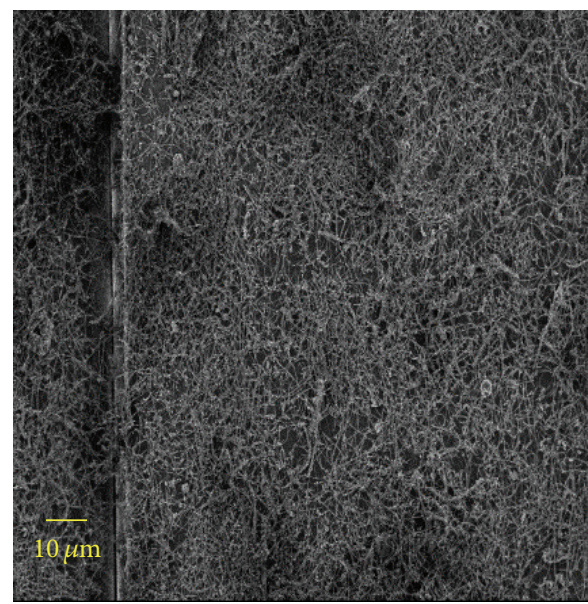

(a)

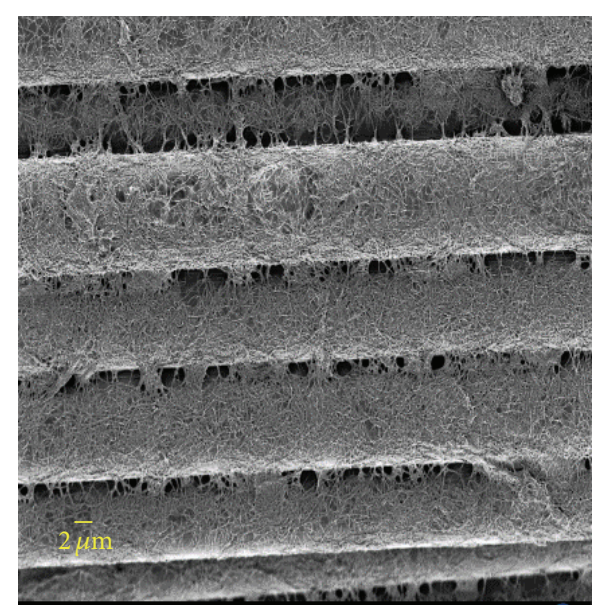

(b)

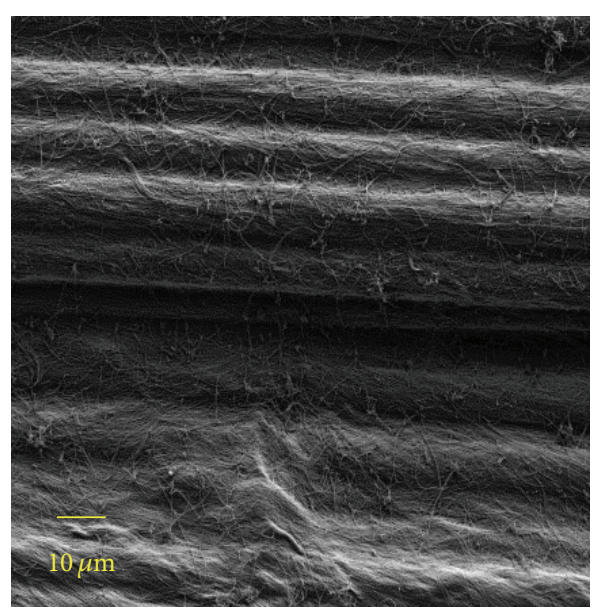

(c)

FIGURE 1: SEM images of carbon fibers coated with (a) MWCNTs, (b) laser SWCNTs, and (c) spun MWCNTs in areas with good coverage. In other areas the fibers are not coated as well, leaving fibers highly exposed.

nanotube containing panels exhibited regions with infinitely high resistivity. This observation is attributed to the CNT dispersion within the composite. When the CNT dispersion is sufficient to form a percolation network between probe heads the resistivity is significantly lower. Otherwise, locations with insufficient CNT concentration result in high resistivity. The regions with low resistivity would be indicative of the level of resistivity that would be achieved if uniform CNT dispersion was accomplished and for this reason the lowest value was reported. It is expected that better CNT dispersion throughout the matrix and higher loadings will result in more consistent conductivity throughout the composite.

In $T_{g}$ measurements obtained by DSC, some samples exhibited two endothermic events in the first thermal cycle of the measurement, while only one was observed in the second thermal cycle (Figure 3 ). The disappearance of the first endothermic event was attributed to potential outgassing of residual solvent used in composite fabrication. Thermogravimetric analysis showed a $3-5 \%$ mass loss at approximately $70^{\circ} \mathrm{C}$. Therefore, the second cycle was used for attainment of $T_{g}$ values of all composites. A shift in the $T_{g}$ to lower temperature was typically observed in the second endothermal event. When the $T_{g}$ of all composites were compared, a $20^{\circ} \mathrm{C}$ decrease is observed in the $T_{g}$ of composites panels 9 and 10 relative to the control. CNTs have been observed to have effects on the $T_{g}$ of polymer-based composites, shifting $T_{g}$ to both higher [25-27] and lower temperatures [28-30]. For these composites, the observations are not clearly understood, but one possible explanation is that Diels Alder chemistry is occurring between the furan or maleimide groups and the delocalized electron network of the CNTs. It has been shown that Diels Alder chemistry can be used to directly bond to the side wall of CNTs [31-33], and therefore it is proposed that this could be contributing to the shift in the $T_{g}$. Chang and Liu showed that furfuryl alcohol and N-(4-hydroxy-phenyl) maleimide react with MWCNTs at a temperature of $50^{\circ} \mathrm{C}$. It is speculated that similar reactivity may be occurring within these composite materials thus 


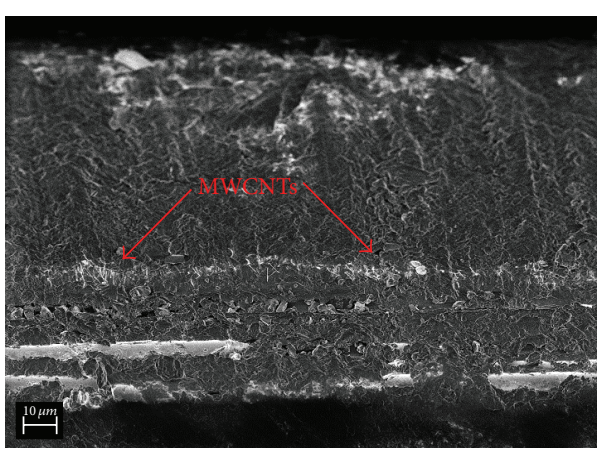

(a)

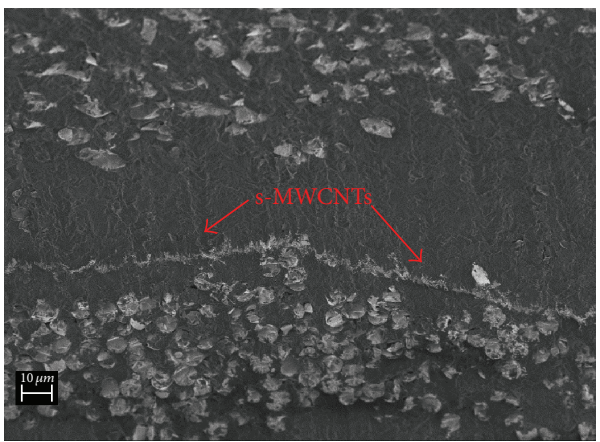

(c)

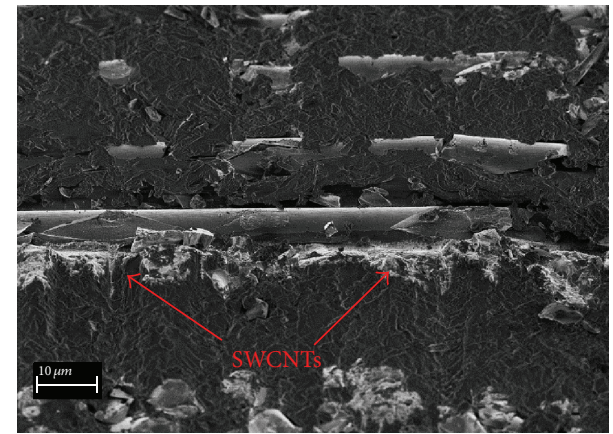

(b)

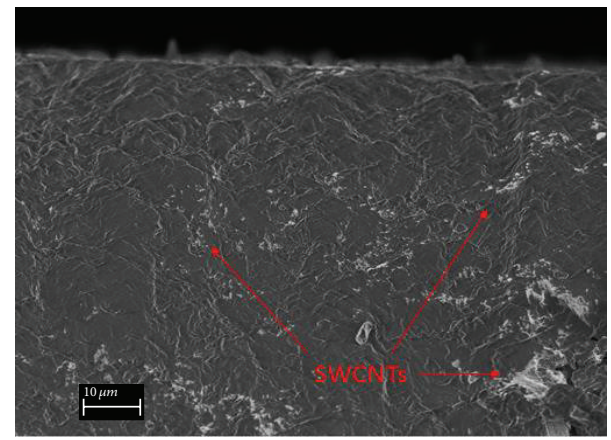

(d)

FIGURE 2: SEM images of various cross-sectioned composite panels showing the location of CNTs within the polymer matrix. CNTs are seen as bright regions within the matrix. Panels with MWCNT spray-coated fibers (a) show greater migration into matrix and extend through to the panel surface. Panels with spun MWCNTs (b) and laser SWCNT sprayed carbon fiber fabrics (c) show lower migration and remain in closer proximity to the fibers. Panels with SWCNTs directly dispersed into the resin during fabrication show mixed behavior with the SWCNTs aggregating within the matrix (d).

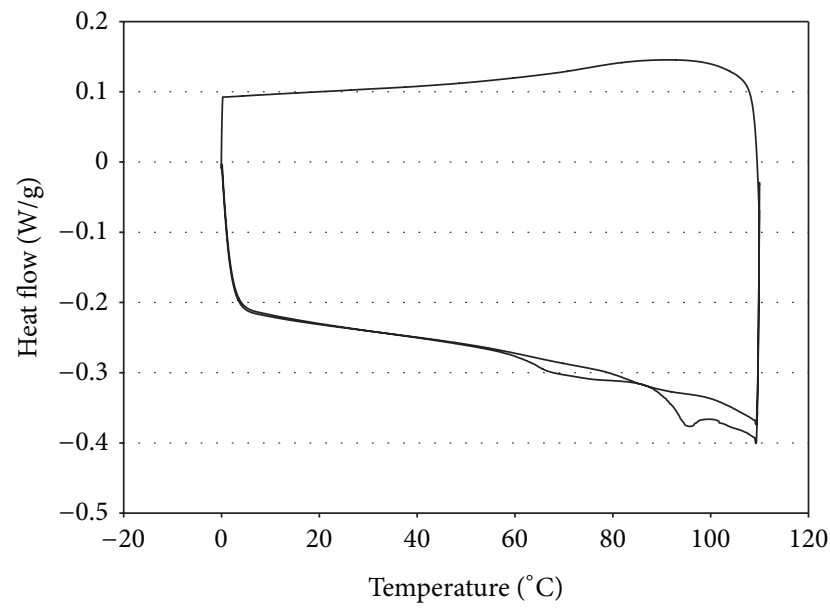

FIGURE 3: Differential scanning calorimetry of panel 7 showing two distinct transitions in the first cycle while only one occurs in the second cycle.

attributing to the lower observed $T_{g}$ values for panels 10 and 11. Further studies are underway to establish if this is indeed contributing to the observed $T_{g}$ depression.

For every composite containing CNTs, the $C_{p}$ shows an increase as seen in Figure 4. It was observed that panels with dispersed SWCNTs had the highest $C_{p}$ s. For other
CNT composites the $C_{p}$ values were lower when greater variation in measurements was observed (shown by data points of panel 8 in Figure 4). Perhaps two phenomena may be influencing the observed increases in $C_{p}$. First, the CNTs may facilitate bond dissociation of the resin at elevated temperatures near the nanotube/resin interface. The accelerated increase in heat capacity above $T_{g}$ is proposed to be supportive of this hypothesis. At this point, thermal energy is being consumed for bond dissociation, resulting in a more rapid increase of the $C_{p}$ beyond the $T_{g}$. Another possible contribution to increases in $C_{p}$ may be attributed to in-plane thermal conduction. As the sample is heating, the thermal energy is transported orthogonally to the thickness, requiring more thermal energy to raise the panel temperature. While a precise description of the physical phenomena requires further investigation, it is evident that CNTs influence both the $T_{g}$ and $C_{p}$ of the composites.

The thermal expansion of the composite is required in order to assess the thermal conductivity. The average dimensional change of the three different composites over the temperature range of $30-110^{\circ} \mathrm{C}$ is shown in Figure 5. The composites with SWCNTs directly dispersed into the matrix show greater expansion relative to the control panel. At $110^{\circ} \mathrm{C}$ the dimensional change increases by approximately $50 \%$. On the other hand, composites consisting of spun CNTs and spray-coated laser SWCNTs show a decrease in expansion 


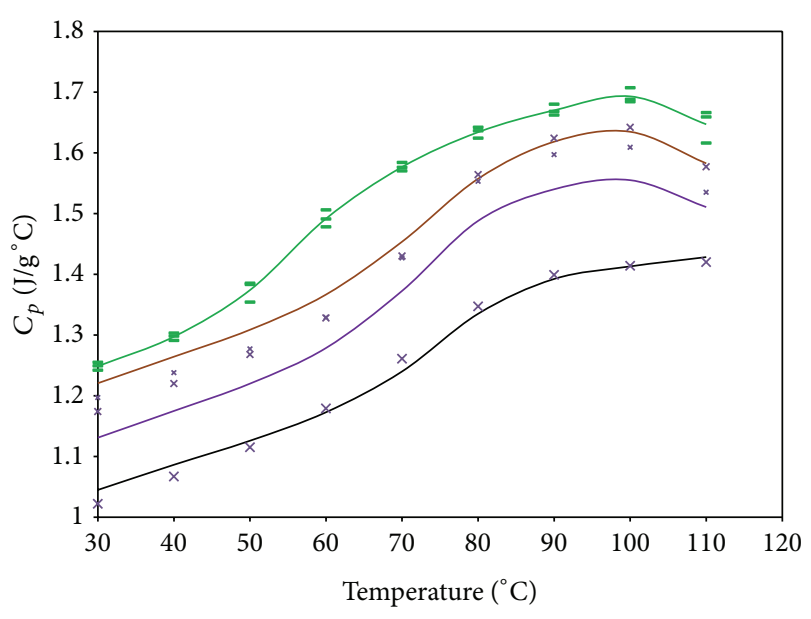

FIGURE 4: Heat capacity curves for panel 1 (black), panel 4 (brown), panel 8 (purple), and panel 9 (green), indicating increases with the addition of CNTs. Glass transitions shifts are evident and the slope is steeper in panel 9 which is believed to be associated with accelerated bond dissociation.

with respect to the control panel. Other composites consisting of fiber fabrics spray-coated with CNTs display expansions comparable to the control. The lower expansion of several of the composites is attributed to anisotropic expansion due to the location of CNTs within the composite. In composites with nanotubes located in close proximity to the fibers, the expansion across the thickness of the panel is lower. It is believed that, in these panels, the in-plane expansion exceeds the out-of-plane expansion, resulting in the lower observed expansion rate. Unfortunately, the in-plane expansion could not be measured because the composite thickness was not sufficient to allow for measurement by the TMA probe. In panels with greater CNT migration into the matrix, the expansion is greater relative to the control. Again, it is speculated that the CNTs may be interacting with the polymer matrix so as to facilitate Diels Alder bond dissociation and thereby cause localized polymer movement. If this were the case, it would be expected that in composites where the CNTs are in closer proximity to the fiber, expansion would be greater in the in-plane direction. On the other hand, in composite where CNTs migrate deeper into the matrix, the through-thickness expansion of the composite is expected to be greater. When the three individual runs for each composite are compared, statistical variation in the expansion measurements is observed to be much like that seen in the $C_{p}$. This variation is attributed to disparity in the concentration of CNTs within the sample. In the case of panel 7 when CNT concentration is low the expansion is almost identical to the control. When concentration is higher, the expansion is almost half that of the control. This observation gives support to the speculation that nanotube resin interaction may be occurring; however, further studies are required to confirm this hypothesis.

Thermal conductivities of composites are represented in Table 1. The conductivities of composites consisting of dispersed SWCNTs in the matrix exhibit lower values as

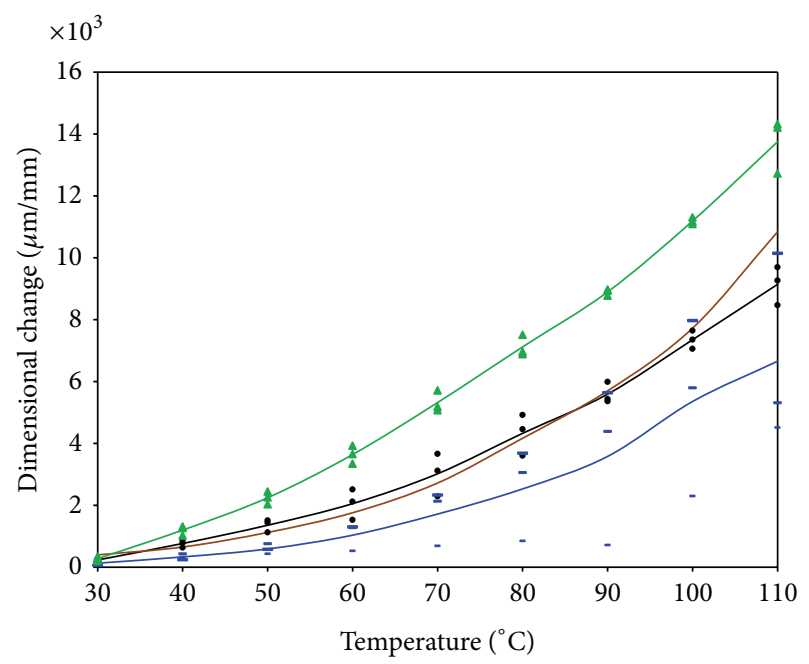

FIGURE 5: Thermal mechanical analysis data showing thermal expansion of panel 1 (black), panel 4 (brown), panel 7 (blue), and panel 9 (green). Lower thermal expansion value of panel 7 is attributed to greater expansion in the in-plane direction as opposed to the out-of-plane direction that is measured. When CNT concentration is low composite expansion is similar to control as seen by panel 7 data points (-). Higher thermal expansion is ascribed to greater bond dissociation.

opposed to those with spray-coated nanoadditives or spun CNTs. Ideally, the excellent thermal properties of CNTs [3438] would be expected to increase the diffusivity and thus the conductivity of the composites. The lower value in conductivity of SWCNT dispersed composites is thought to be the result of the greater thermal expansion observed in TMA. The heat imparted by the laser flash must traverse across a greater length and therefore the observed diffusivity is likely lowered. Likewise, panels with lower thermal expansion exhibit higher diffusivity values for the opposite reason. In addition to the lower diffusivity in SWCNT dispersed panels, the data show more scatter over the entire temperature range of collection. Again, this is attributed to the variability in the dispersion of SWCNT within the various samples.

The thermal conductivity as a function of temperature of several composites panels are shown in Figure 6. It is clear that improvement is witnessed across the board as would be expected. While the increase of $55 \%$ in thermal conductivity may be viewed as relatively modest, it would be anticipated that a greater increase will be achieved through optimization of CNT dispersion and minimization of thermal expansion. Optimization of CNT dispersion will allow for a greater percolation threshold for conductivity to be achieved. Studies are underway to enhance the dispersion of CNT throughout the $2 \mathrm{MEP} 4 \mathrm{~F}$ resin by controlled functionalization of CNTs. This could also reduce expansion by direct bonding of the CNTs to the polymer and thus potentially locally constraining polymer movement.

The thermomechanical properties of composites were measured as described above. The storage and loss modulus were compared for all composite samples. The storage moduli at $30^{\circ} \mathrm{C}$ are reported in Table 1 . This temperature was used 


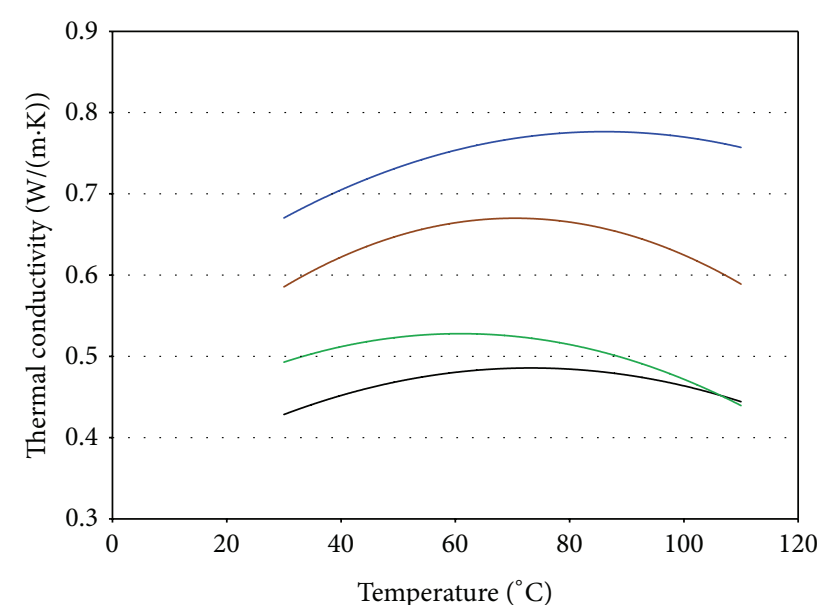

FIGURE 6: Thermal conductivity measurement of panel 1 (black), panel 4 (brown), panel 7 (blue), and panel 9 (green). Panel 9 shows decreasing conductivity with temperature, attributed to changes in diffusivity resulting from volume expansion and bond dissociation.

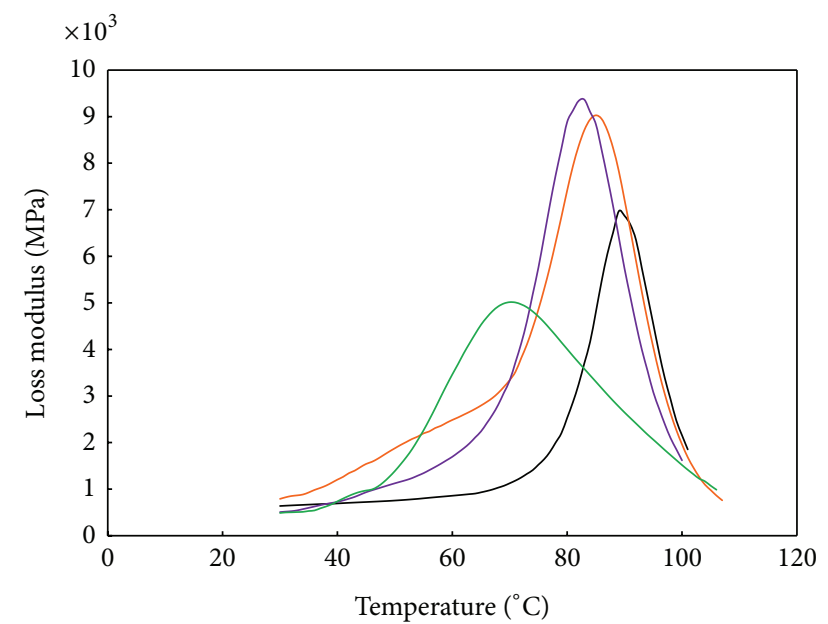

FIgURE 7: Loss moduli of composite panel 1 (black), panel 6 (orange), panel 8 (purple), and panel 9 (green). Shifts and broadening of the peaks are attributed to polymer chain rearrangement and bond dissociation.

to give an indication of the inherent mechanical properties of the composite near room temperature. It is clear that the storage modulus increases for CNT-containing panels. This translates to higher elasticity within the materials and thus higher stiffness. The peak maximum in the loss moduli and corresponding temperatures are also reported. The loss moduli are associated with energy dissipated as heat. Shifts in the loss moduli peak positions to lower temperatures (Figure 7) are seen and are interpreted as either polymer rearrangement or potential bond dissociation at these lower temperatures. The loss modulus also broadens. This may indicate that better nanotube dispersion results in greater polymer nanotube interaction over a broader temperature range.
Composite panels were impacted with imposing energies of $1.15 \mathrm{~J}$ and $2.25 \mathrm{~J}$. The impacter was not capable of measuring deflection at the time of the testing, so a quantitative measurement of toughness has not been made to date. Qualitative measurement of toughness was evaluated by electron microscopy. Figure 8 shows the control panel and a nanotube containing panel, both subjected to the same drop force. It is evident that less fiber exposure is observed and the polymer resin remains more intact in nanotube containing composites. This is due to the increased strength and toughness imparted by the CNTs.

Repolymerization of the $2 \mathrm{MEP} 4 \mathrm{~F}$ resin is induced by thermal activation in the form of microwave heating. It is therefore important to understand the influence of CNTs on the microwave absorption behavior and the heating efficiency of the composite. The composite must have adequate microwave absorption in order to achieve the target thermalreversible initiation temperature of $110-120^{\circ} \mathrm{C}$ in a reasonable period of time. Furthermore, it is desirable to have a uniform thermal distribution over the entire area of the composite so that the composite heals at the same rate throughout the structure.

To explore the heating properties, several composite panels were exposed to microwaves of various powers while monitoring the panels' surface temperatures with an IR camera. Figure 9 shows the surface temperature profiles of three composite panels exposed to microwaves at the same power of 300 watts. After 22 minutes of exposure, panel 1 (control sample) shows localized heating at the edges, likely due to protruding carbon fiber at these locations. Thermal distribution is poor as it is expected that the embedded fibers have lower absorption and thermal conduction. The addition of CNTs improves the heating efficiency of the composites as evidenced by the greater uniformity of the surface temperature and shorter exposure times of 10 minutes required to reach higher temperatures. It is evident that both SWCNTs and MWCNTs improve the heating capability of the composite. A qualitative assessment of the panels' relative heating efficiencies is then made from these thermal profiles. A higher heating efficiency is ascribed to a panel that requires lower microwave power to reach the same target temperature in the same amount of time as another. On the other hand, when subjected to the same power level, a shorter exposure time is also interpreted as better heating efficiency. Microwave exposures assessed in this manner were used to qualitatively compare the influence of CNTs on the heating efficiency of the composite panels.

The best heating efficiency is observed with spun CNTs deposited on the carbon fiber fabrics. This is attributed to the higher density of nanotubes and greater continuity between nanotubes achieved during the spinning process. Follow-on studies will include microwave exposure of panels consisting of fiber fabrics with spun CNTs directly applied and with SWCNTs dispersed in the matrix. It is expected that these panels will show further improved heating efficiency due to better distribution of nanotubes throughout the composites, thus improving the percolation threshold and giving greater thermal continuity throughout the matrix. Since the CNTs serve as a local heat source during microwave exposure, it 


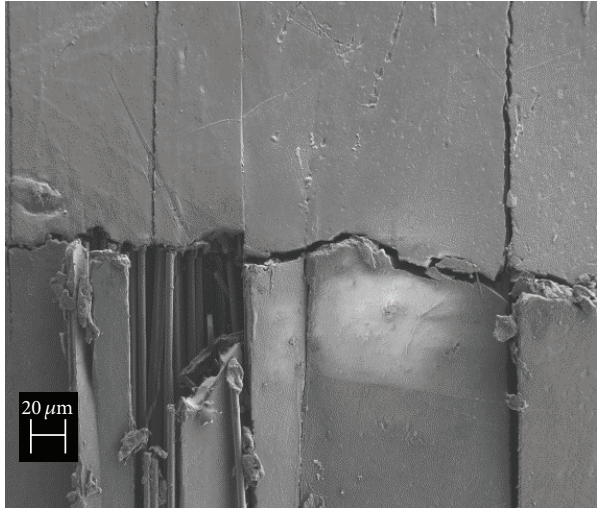

(a)

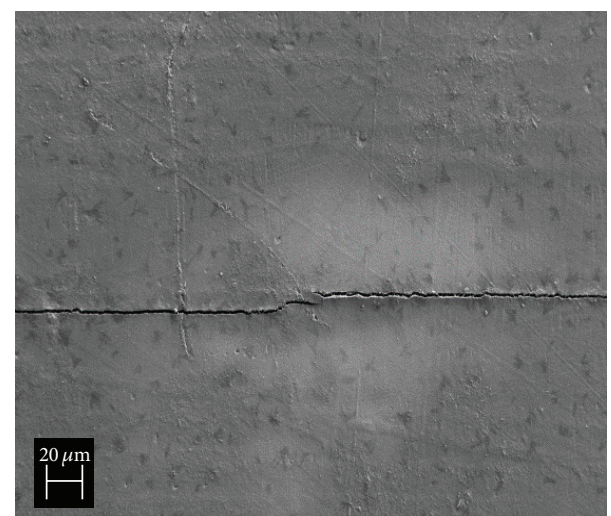

(b)

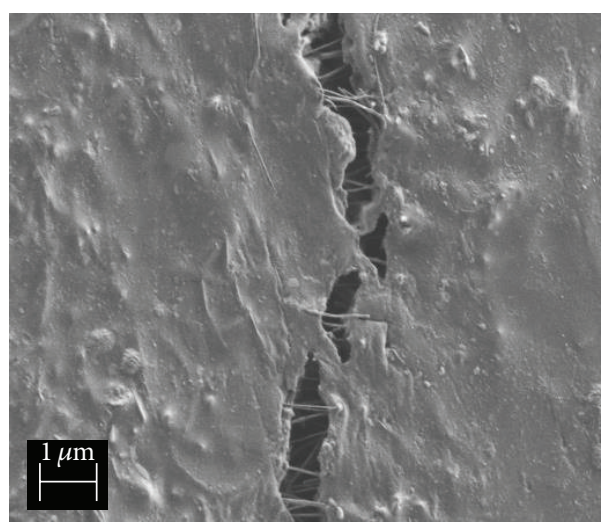

(c)

FIGURE 8: Scanning electron images of impacted composite panel showing the differences in the degree of damage between a control panel without CNTs (a) and one with MWCNT-coated fiber fabrics (b). It is presumed that the matrix is held more intact due to the load bearing property of CNTs as demonstrated by crack bridging (c).

is expected that small-scale, localized thermal gradients will exist throughout the composite due to differences between the thermal conductivity of the polymer and the CNTs. Ideal CNT dispersion will help minimize this effect and allow for better translation of heat through the composite. This can assist in the healing of damage located deeper within the interior of the composite. Although these localized thermal gradients may exist, the goal is to reach a steady-state temperature for healing and thus it is important to achieve a uniform thermal distribution. Therefore, follow-on studies will more quantitatively investigate the influence of nanotube loading on both heating and healing efficacy.

In addition to as-fabricated panels, two CNT-containing, impacted panels were also exposed to microwave irradiation. Figure 10 shows thermal profiles of their surface. It is clearly evident that local hot spots appear in the areas of impaction. Figure $8(\mathrm{c})$ shows microcrack formations that are produced upon impact. CNTs can be seen bridging the width of such cracks. These exposed CNTs are directly exposed to the microwaves and therefore generate heat more rapidly than the embedded CNTs, resulting in these hot spots. The observation of these localized hot spots suggests a means for damage detection and could potentially allow monitoring of the healing process. During the healing process, it is expected that the resin will mend the cracks, reembed the CNTs, and result in the disappearance of the hot spot. Such an observation would allow for real-time structural health monitoring of the composite structure. Forward work includes these studies through longer term microwave heating and thermal imaging of composites.

\section{Conclusion}

The inclusion of various CNT materials into self-healing polymer resin/carbon fiber composites has been shown to introduce multifunctional properties to the composites. Carbon fiber fabrics were coated with a variety of carbon nanomaterials for panel fabrication. The electrical, thermal, and mechanical properties are enhanced through the introduction of various CNTs, and while being modest in some cases, it is clear that improvements can be achieved. The electrical resistivity is reduced by six orders of magnitude from giga-ohm to kilo-ohm with substantial variations in the resistivity across the composites.

The thermal properties of the composites are significantly influenced by the presence of carbon nanoadditives. The thermal conductivity increases for all composites containing nanoadditives. The largest increase of $55 \%$ is seen in the 


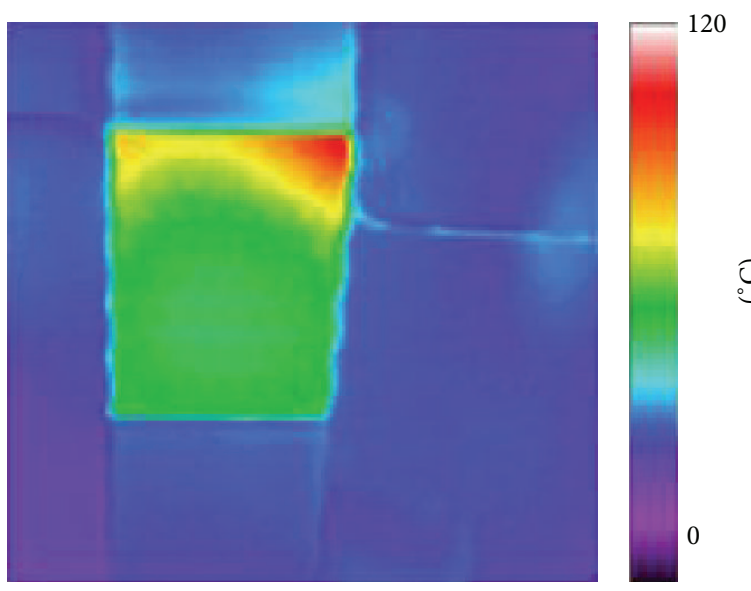

(a)

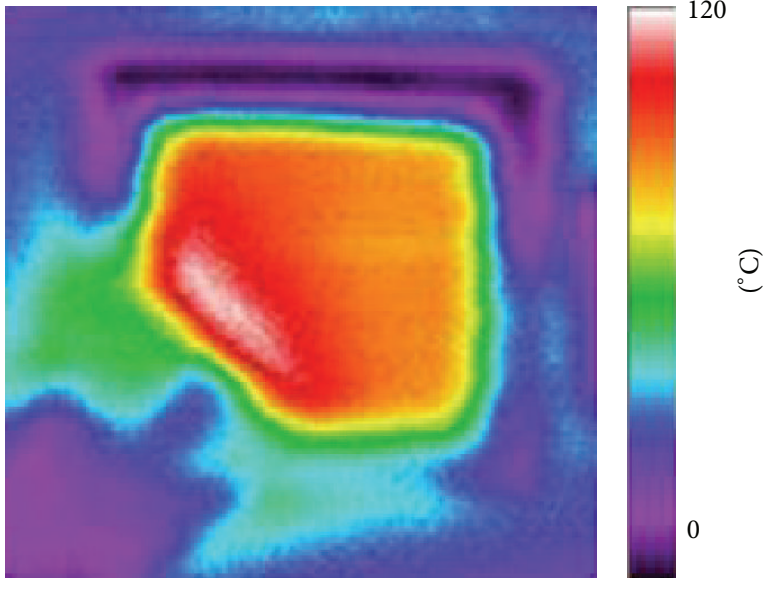

(b)

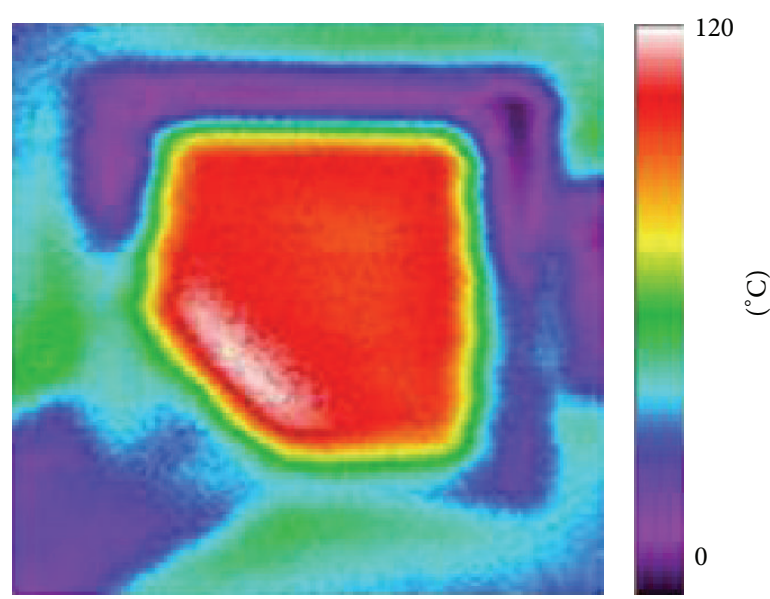

(c)

Figure 9: Thermal imaging of composite panel 1 (a), panel 6 (b), and panel 7 (c) under microwave exposure. Panel 1 is a $10 \mathrm{~cm} \times 10 \mathrm{~cm}$ composite but appears oblong due to orientation of thermal camera. Panels 6 and 7 are $5 \mathrm{~cm} \times 5 \mathrm{~cm}$ composites that were masked along the edges with microwave nonabsorbing tape to prevent arching along edges. It is clear that introduction of CNTs allows for better heating efficiency as the entire panel can be heated to the required temperature for healing.

composite panels containing oriented spun MWCNTs. The heat capacities of all carbon nanoadditive composites are increased while the glass transitions remain quite similar with the exception of two of the panels. The glass transition temperature of the two composite panels where SWCNTs were directly dispersed into the resin prior to lay up shows a $20^{\circ}$ decrease. The thermal expansion of the SWCNT dispersed composites increase by as much as $50 \%$ while the composite with s-MWCNT-coated carbon fibers shows $27 \%$ decrease relative to the control sample. It is speculated that lowering of the glass transition and disparity of the thermal expansions could be the result of direct interaction between furan and maleimide groups with the sidewalls of the SWCNTs. Forward work includes determining if covalent bonding occurs between the CNTs and resin that contributes to the lowering of the glass transition and changes in thermal expansions.

CNTs were also shown to improve the mechanical integrity of the composite. Impacted nanotube containing panels show less extensive fiber pull-out and lower loss of the matrix. Composite panels containing CNTs undergo less extensive damage when impacted due to crack bridging and load transfer. The storage modulus increases upon the introduction of CNTs, indicating greater elasticity. This supports the qualitative observation made of the damage morphology of impacted samples and is expected to translate to higher strength.

Exposure of several composite panels to microwave energy shows that composites can be uniformly heated to the desired temperature to allow thermally reversible polymerization. Furthermore, microwave irradiation of impacted panels distinctively display localized heat generation in the area of damage. This observation provides a means for damage detection. It is proposed that this could potentially be used to monitor the healing process within the composite thereby introducing real-time health monitoring. Development of multifunctional composites capable of self-repair, damage inspection, and real-time health monitoring could 


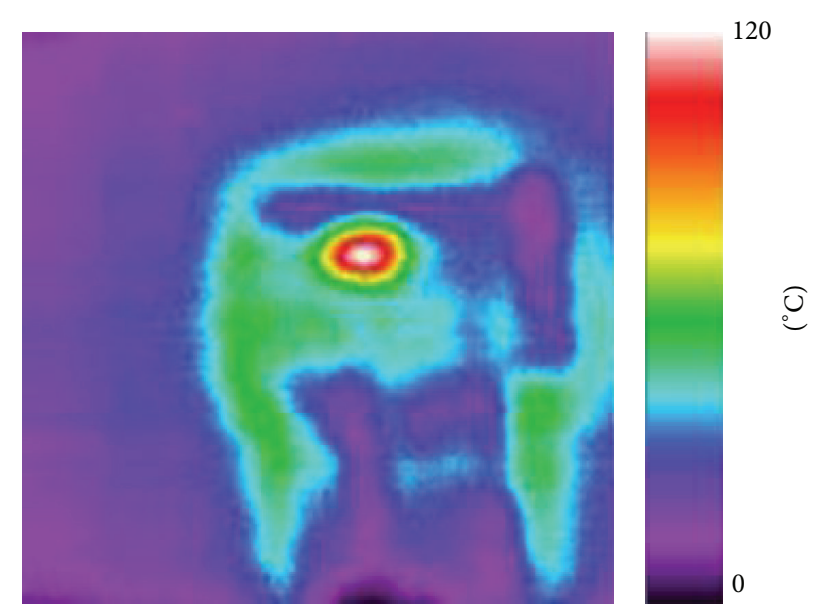

FIGURE 10: Thermal imaging of an impacted $5 \mathrm{~cm} \times 5 \mathrm{~cm}$ panel while under microwave irradiation shows a local hot spot in the area of damage. It is proposed that this property could be exploited as a means for damage inspection and structural health monitoring.

result in more efficient, safe, and reliable long duration space exploration.

\section{Conflict of Interests}

The authors declare that there is no conflict of interests regarding the publication of this paper.

\section{Acknowledgments}

The authors would like to thank Dr. Anvar Zhakidov, Dr. John Ferraris, and William Holmes at Solarno for deposition of spun carbon nanotubes onto carbon fibers. Dr. Mark Sanders from the University of Minnesota is also acknowledged for microwave testing on composite panels. This work was funded through the NASA Center Innovation Fund directed by the Johnson Space Center towards Independent Research and Development. This paper is based on work supported by the Air Force Office of Scientific Research Contract FA9550-11-C-0008. Appreciation is extended to Dr. B. Les Lee, Program Manager, for his encouragement and support.

\section{References}

[1] D. Y. Wu, S. Meure, and D. Solomon, "Self-healing polymeric materials: a review of recent developments," Progress in Polymer Science, vol. 33, no. 5, pp. 479-522, 2008.

[2] S. D. Bergman and F. Wudl, "Mendable polymers," Journal of Materials Chemistry, vol. 18, no. 1, pp. 41-62, 2008.

[3] M. R. Kessler, "Self-healing: a new paradigm in materials design," Proceedings of the Institution of Mechanical Engineers G: Journal of Aerospace Engineering, vol. 221, no. 4, pp. 479-495, 2007.

[4] J. W. C. Pang and I. P. Bond, "A hollow fibre reinforced polymer composite encompassing self-healing and enhanced damage visibility," Composites Science and Technology, vol. 65, no. 11-12, pp. 1791-1799, 2005.
[5] B. J. Blaiszik, M. M. Caruso, D. A. Mcllroy, J. S. Moore, S. R. White, and N. R. Sottos, "Microcapsules filled with reactive solutions for self-healing materials," Polymer, vol. 50, no. 4, pp. 990-997, 2009.

[6] J. Hu, H.-Q. Chen, and Z. Zhang, "Mechanical properties of melamine formaldehyde microcapsules for self-healing materials," Materials Chemistry and Physics, vol. 118, no. 1, pp. 63-70, 2009.

[7] T. F. Scott, A. D. Schneider, W. D. Cook, and C. N. Bowman, "Photoinduced plasticity in cross-linked polymers," Science, vol. 308, no. 5728, pp. 1615-1617, 2005.

[8] Y. Amamoto, J. Kamada, H. Otsuka, A. Takahara, and K. Matyjaszewski, "Repeatable photoinduced self-healing of covalently cross-linked polymers through reshuffling of trithiocarbonate units," Angewandte Chemie, vol. 50, no. 7, pp. 1660-1663, 2011.

[9] M. E. Garcia, Y. Lin, and H. A. Sodano, "Autonomous materials with controlled toughening and healing," Journal of Applied Physics, vol. 108, no. 9, Article ID 093512, 2010.

[10] F. Ghezzo, D. R. Smith, T. N. Starr et al., "Development and characterization of healable carbon fiber composites with a reversibly cross linked polymer," Journal of Composite Materials, vol. 44, no. 13, pp. 1587-1603, 2010.

[11] X. Chen, F. Wudl, A. K. Mal, H. Shen, and S. R. Nutt, "New thermally remendable highly cross-linked polymeric materials," Macromolecules, vol. 36, no. 6, pp. 1802-1807, 2003.

[12] T. A. Plaisted and S. Nemat-Nasser, "Quantitative evaluation of fracture, healing and re-healing of a reversibly cross-linked polymer," Acta Materialia, vol. 55, no. 17, pp. 5684-5696, 2007.

[13] M. L. Szalai, D. V. McGrath, D. R. Wheeler, T. Zifer, and J. R. McElhanon, "Dendrimers based on thermally reversible furanmaleimide Diels-Alder adducts," Macromolecules, vol. 40, no. 4, pp. 818-823, 2007.

[14] J. S. Park, T. Darlington, A. F. Starr, K. Takahashi, J. Riendeau, and $\mathrm{H}$. Thomas Hahn, "Multiple healing effect of thermally activated self-healing composites based on Diels-Alder reaction," Composites Science and Technology, vol. 70, no. 15, pp. 21542159, 2010.

[15] J. C. J. Bart, Additives in Polymers: Industrial Analysis and Applications, John Wiley \& Sons, New York, NY, USA, 2005.

[16] T. J. Imholt, C. A. Dyke, B. Hasslacher et al., " Nanotubes in microwave fields: light emission, intense heat, outgassing and reconstruction," Chemistry of Materials, vol. 15, no. 21, pp. 39693970, 2003.

[17] P. Zhihua, P. Jingcui, P. Yanfeng, O. Yangyu, and N. Yantao, "Investigation of the microwave absorbing mechanisms of HiPco carbon nanotubes," Physica E, vol. 40, no. 7, pp. 24002405, 2008.

[18] K. R. Paton and A. H. Windle, "Efficient microwave energy absorption by carbon nanotubes," Carbon, vol. 46, no. 14, pp. 1935-1941, 2008.

[19] J. A. Menéndez, A. Arenillas, B. Fidalgo et al., "Microwave heating processes involving carbon materials," Fuel Processing Technology, vol. 91, no. 1, pp. 1-8, 2010.

[20] A. L. Higginbotham, P. G. Moloney, M. C. Waid et al., "Carbon nanotube composite curing through absorption of microwave radiation," Composites Science and Technology, vol. 68, no. 1516, pp. 3087-3092, 2008.

[21] R. G. de Villoria, N. Yamamoto, A. Miravete, and B. L. Wardle, "Multi-physics damage sensing in nano-engineered structural composites," Nanotechnology, vol. 22, no. 18, Article ID 185502, 2011. 
[22] R. G. de Villoria, N. Yamamoto, A. Miravete, and B. L. Wardle, "Purification and characterization of single-wall carbon nanotubes," Journal of Physical Chemistry B, vol. 105, no. 6, pp. 11571161, 2001.

[23] P. Nikolaev, O. Gorelik, R. K. Allada, E. Sosa, S. Arepalli, and L. Yowell, "Soft-bake purification of single-walled carbon nanotubes produced by pulsed laser vaporization," Journal of Physical Chemistry C, vol. 111, no. 48, pp. 17678-17683, 2007.

[24] L. Yowell, P. Nikolaev, O. Gorelik, R. K. Allada, E. Sosa, and S. Arepalli, "Soft-Bake purification of SWCNTs produced by pulsed laser vaporization,” NASA TechBriefs, 2013.

[25] T. Sterzyński, J. Tomaszewska, K. Piszczek, and K. Skórczewska, "The influence of carbon nanotubes on the PVC glass transition temperature," Composites Science and Technology, vol. 70, no. 6, pp. 966-969, 2010.

[26] M. Farbod and S. Khajehpour Tadavani, "Electrical properties and glass transition temperature of multiwalled carbon nanotube/polyaniline composites," Journal of Non-Crystalline Solids, vol. 358, no. 11, pp. 1339-1344, 2012.

[27] A. Warrier, A. Godara, O. Rochez et al., "The effect of adding carbon nanotubes to glass/epoxy composites in the fibre sizing and/or the matrix," Composites A: Applied Science and Manufacturing, vol. 41, no. 4, pp. 532-538, 2010.

[28] F. Y. Castillo, R. Socher, B. Krause et al., "Electrical, mechanical, and glass transition behavior of polycarbonate-based nanocomposites with different multi-walled carbon nanotubes," Polymer, vol. 52, no. 17, pp. 3835-3845, 2011.

[29] X. Yan, Z. Gong, J. Gong, S. Gao, B. Wang, and X. Ruan, "Investigation of the glass transition and viscoelastic properties of polycarbonate/multi-walled carbon nanotube composites by positron annihilation lifetime spectroscopy," Polymer, vol. 54, no. 2, pp. 798-804, 2013.

[30] X. Wang, H. Liu, and L. Qiu, "Cationic polymerization of tetrahydrofuran from multiple-walled carbon nanotubes: preparation and glass transition kinetics," Materials Letters, vol. 61, no. 11-12, pp. 2350-2353, 2007.

[31] C. Chang and Y. Liu, "Functionalization of multi-walled carbon nanotubes with furan and maleimide compounds through Diels-Alder cycloaddition," Carbon, vol. 47, no. 13, pp. 30413049, 2009.

[32] M. T. Beck, J. Szépvölgyi, P. Szabó, and E. Jakab, "Heterogeneous DielsAlder reaction between cyclopentadiene and different solid carbons," Carbon, vol. 39, pp. 137-158, 2001.

[33] E. H. Fort and L. T. Scott, "Gas-phase Diels-Alder cycloaddition of benzyne to an aromatic hydrocarbon bay region: groundwork for the selective solvent-free growth of armchair carbon nanotubes," Tetrahedron Letters, vol. 52, no. 17, pp. 2051-2053, 2011.

[34] J. Che, T. Çağin, and W. A. Goddard III, “Thermal conductivity of carbon nanotubes," Nanotechnology, vol. 11, no. 2, pp. 65-69, 2000.

[35] J. Hone, M. C. Llaguno, N. M. Nemes et al., "Electrical and thermal transport properties of magnetically aligned single wall carbon nanotube films," Applied Physics Letters, vol. 77, no. 5, pp. 666-668, 2000.

[36] J. Hone, M. Whitney, C. Piskoti, and A. Zettl, “Thermal conductivity of single-walled carbon nanotubes," Physical Review B, vol. 59, no. 4, pp. R2514-R2516, 1999.

[37] W. Zhang, Z. Zhu, F. Wang, T. Wang, L. Sun, and Z. Wang, "Chirality dependence of the thermal conductivity of carbon nanotubes," Nanotechnology, vol. 15, no. 8, pp. 936-939, 2004.
[38] P. Kim, L. Shi, A. Majumdar, and P. L. McEuen, "Thermal transport measurements of individual multiwalled nanotubes," Physical Review Letters, vol. 87, no. 21, Article ID 215502, 2001. 

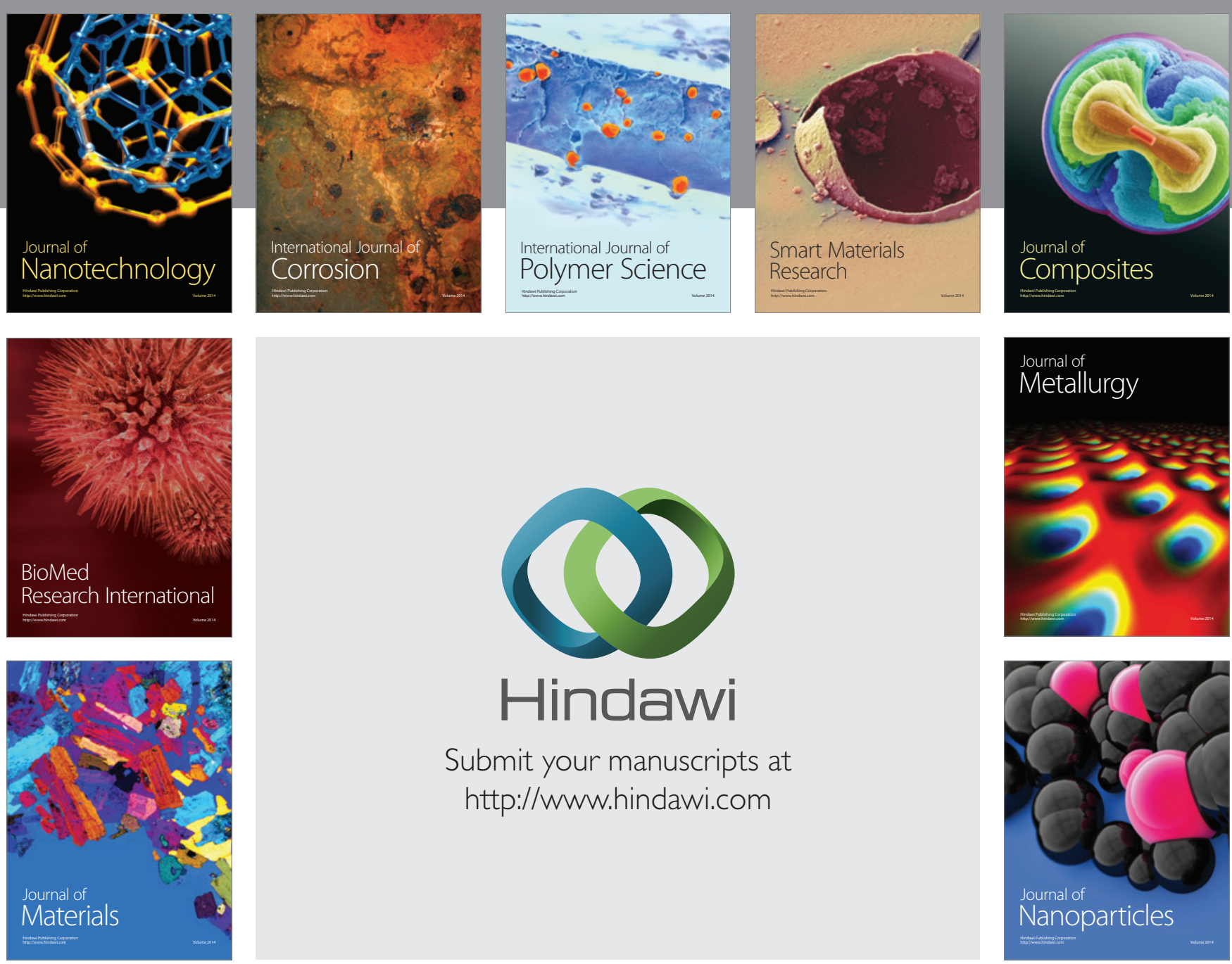

Submit your manuscripts at http://www.hindawi.com
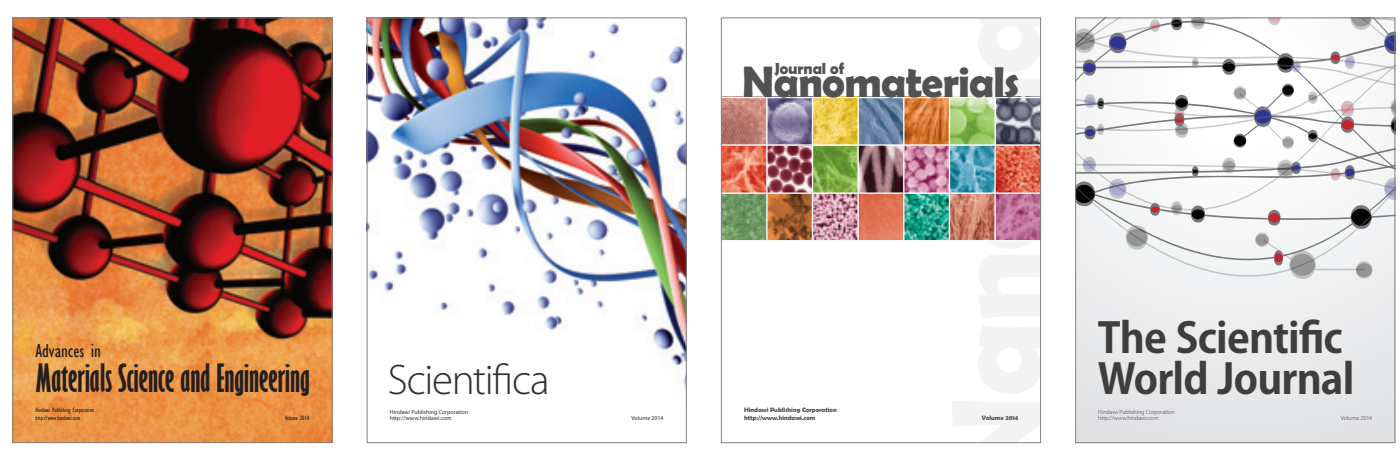

\section{The Scientific World Journal}
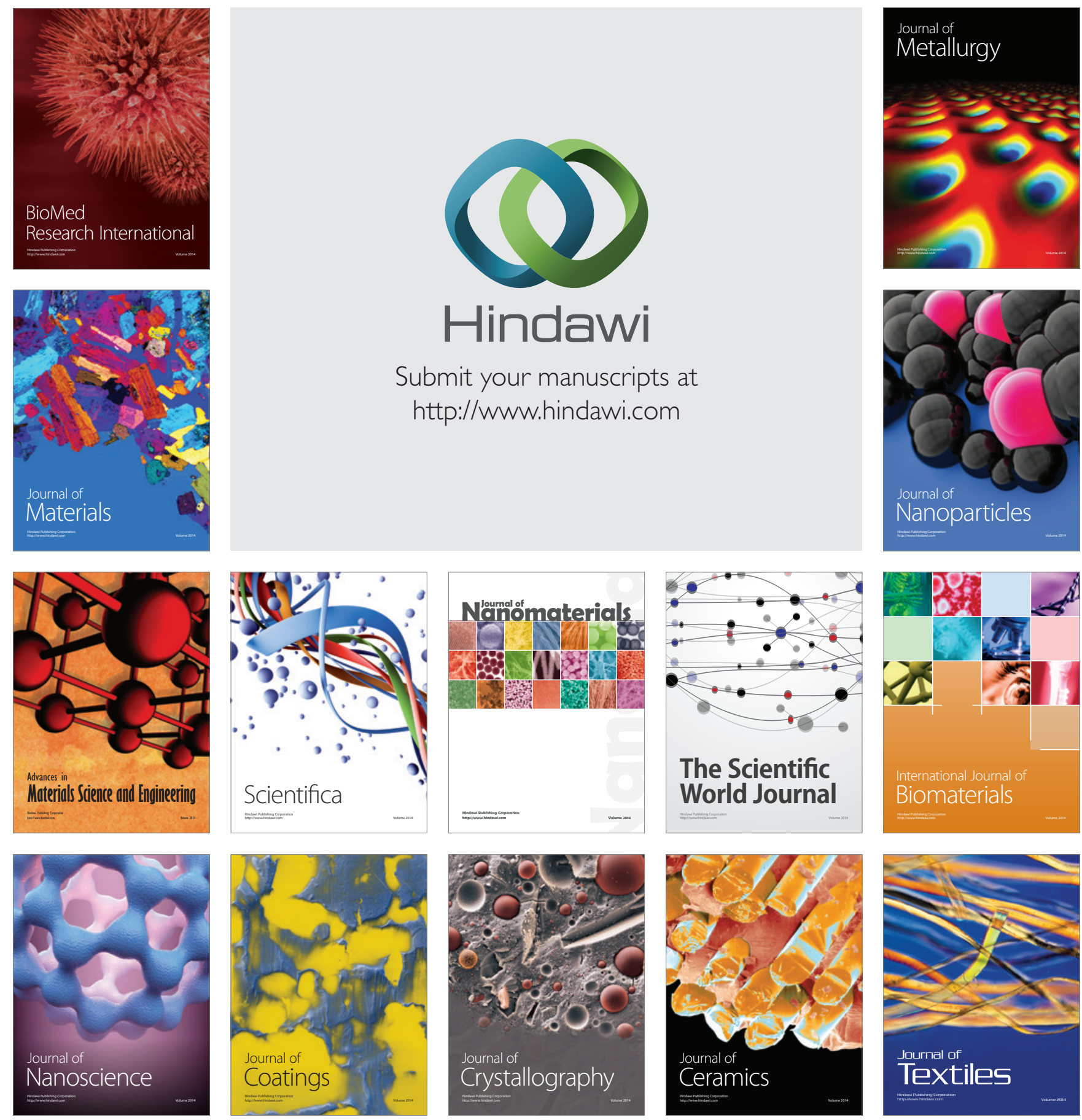